\title{
Marginal Zone B-Lymphocyte of Nodal Type
}

National Cancer Institute

\section{Source}

National Cancer Institute. Marginal Zone B-Lymphocyte of Nodal Type. NCI Thesaurus. Code C38322.

A lymphocyte found in the marginal zones of lymph nodes. It has a naive B lymphoid lineage and plays an important role in the early phases of immune response with its ability to rapidly differentiate into an antibody secreting cell. These cells can directly activate T cells, interact with other antigen presenting cells, transporting and concentrating antigen during the course of $\mathrm{T}$-dependent and $\mathrm{T}$-independent immune responses. 